\title{
Shining a Light on Haemonchus contortus in Sheep ${ }^{\dagger}$
}

\author{
Elise Kho 1, Jill Fernandes ' , Andrew Kotze 2, Maggy Lord ${ }^{3}$, Glen Fox ${ }^{4}$, Anne Beasley ${ }^{5}$, \\ Stephen Moore ${ }^{1}$ and Peter James ${ }^{1, *}$
}

1 The Queensland Alliance for Agriculture and Food Innovation, The University of Queensland, St. Lucia, Queensland 4067, Australia; elise.kho@uq.edu.au (E.K.); j.ulrich@uq.edu.au (J.F.); s.moore3@uq.edu.au (S.M.)

2 CSIRO Agriculture and Food, St. Lucia, Queensland 4067, Australia; andrew.kotze@csiro.au

3 The School of Public Health, The University of Queensland, Herston, Queensland 4006, Australia; maggy.lord@uq.edu.au

4 Department of Food Science and Technology, University of California, Davis, CA 95616, USA; gpfox@ucdavis.edu

5 The School of Agriculture \& Food Sciences, The University of Queensland, Gatton, Queensland 4343, Australia; a.beasley@uq.edu.au

* Correspondence: p.james1@uq.edu.au

+ Presented at the third International Tropical Agriculture Conference (TROPAG 2019), Brisbane, Australia, 11-13 November 2019.

Published: 2 April 2020

\begin{abstract}
Heavy infestations of the Barber's pole worm, Haemonchus contortus, can cause severe wasting, morbidity and mortality in animals if not promptly treated. The current detection methods for this blood-sucking parasite involve faecal worm egg counts and diagnosis of anaemia, both of which are time consuming and require expertise. As blood is detected in sheep faeces sooner during infection than worm eggs, quantitative evaluation of blood may serve as a sensitive indicator of $H$. contortus infection. Here we investigated the feasibility of rapid estimation of haemoglobin $(\mathrm{Hb})$ in sheep faeces using visible near-infrared spectroscopy. Haemoglobin $(\mathrm{Hb})$ was assessed at various concentrations in moist sheep faeces using portable visible near-infrared (vis-NIR) spectrometers. Calibration models were developed for the region of 400-600 nm, where $\mathrm{Hb}$ absorption bands can be found. Within this wavelength region, $\mathrm{Hb}$ in sheep faeces can be estimated with minimal interference from background moisture $(970 \mathrm{~nm})$ or chlorophyll $(670 \mathrm{~nm})$, suggesting that difference in diets in sheep will have minimal effect on prediction accuracy. Predictions for sheep faeces based on a drenching threshold of $3 \mu \mathrm{g} \mathrm{Hb} / \mathrm{mg}$ faeces showed high levels of accuracy with minimal sample preparation (Sensitivity $=89 \%$, specificity $=80 \%$ ). The success in detecting $\mathrm{Hb}$ in sheep faeces indicates the potential of vis-NIR spectroscopy as a rapid, on-farm diagnostic method for predicting blood in sheep faeces, and timing treatment of $H$. contortus infections.
\end{abstract}

Keywords: Haemonchus contortus; visible-near infrared spectroscopy; portable spectroscopy; gastrointestinal nematode diagnosis; haemoglobin; faeces; NIR

Conflicts of Interest: The authors declare no conflict of interest.

(C) 2020 by the authors. Licensee MDPI, Basel, Switzerland. This article is an open access article distributed under the terms and conditions of the Creative Commons Attribution (CC BY) license (http://creativecommons.org/licenses/by/4.0/). 